\title{
Oblique Reformatting of Multislice Magnetic Resonance Images for Improved Visualization of Coronary Arteries
}

\author{
Nola M. Hylton, William S. Chung, Elias H. Botvinick, Nelson B. Schiller, Phillip Sheldon, and Leon Kaufman
}

\begin{abstract}
A technique for reformatting multislice magnetic resonance images into arbitrary oblique planes has been developed and implemented on a Toshiba MRT-35 (formerly Diasonics MT/S) imaging system (South San Francisco, CA). This method is designed to allow the user to easily define a new plane by marking with a cursor features of interest on two or three different image levels. These features are combined in the resulting oblique image. The reformatted image can have arbitrary angulation and is created with a pixel dimension equivalent to the original data set. Resolution ranges from the original in-plane resolution to the slice thickness, depending on angulation. An improvement in signal-to-noise ratio results from the effective averaging performed by interpolation. This method is optimally used to correct for small variations in alignment, such as the positioning of the intervertebral disks. It can also be used to generate reformatted images at many different angles from a single multislice data set. This method has been applied to the particular problem of improving the presentation of coronary arteries on a conventional set of multislice spin-echo cardiac images by increasing the visible length of individual coronary segments.
\end{abstract}

(C) 1990 by W.B. Saunders Company.

KEY WORDS: magnetic resonance imaging, image processing, computers.

$T^{\text {H }}$ HE INTRINSIC CONTRAST between flowing blood and solid tissue that exists with magnetic resonance imaging (MRI) make it an attractive means of studying vascular anatomy without the need for contrast media. New methods use time-of-flight effects of moving blood or take advantage of velocity-dependent phase changes to encode information into the image. The prospect that these techniques might be extended to image the coronary arteries has many researchers eagerly investigating imaging strategies that may provide diagnostic information about coronary disease. ${ }^{1,2}$ This turns out to

From the Radiologic Imaging Laboratory and the Echocardiography Laboratory, University of California, San Francisco.

Address reprint requests to Nola $M$. Hylton, PhD, Radiologic Imaging Laboratory, University of California, 400 Grandview Dr South, San Francisco, CA 94080.

(C) 1990 by W.B. Saunders Company.

0897-1889/0301-0008\$03.00/0 be a much more challenging problem because of the nature of coronary anatomy, in particular the narrow dimension and tortuousness of the vessels, the pulsatility of the flow, the motion of the heart, and its underlying blood pools. Low spatial resolution and the tomographic imaging format of MRI compared with angiography make the imaging of coronary vessels particularly difficult.

Conventional two-dimensional Fourier transform (2DFT) spin-echo images are acquired in multislice format using a technique of selective excitation for slice isolation and phase and frequency encoding for in-plane spatial differentiation. This method produces images with slice thicknesses up to 10 times the in-plane pixel dimension. Our cardiac imaging sequence is a multislice, multiphase spin-echo technique that generates transaxial images with $10-\mathrm{mm}$ slice thickness, $256 \times 256$-pixel matrix, and $0.95-\mathrm{mm}$ in-plane resolution. Noninterleaved contiguous slices are acquired with no slice gap. Images at five phases of the cardiac cycle are collected using electrocardiographic gating and triggering on the $R$ wave. An echo-delay time (TE) value of 30 milliseconds is used, and the pulse-repetition interval (TR) is determined by the R-R interval. Images are acquired perpendicular to the body axis at 10 levels, roughly spanning the region between the aortic root and apex. This sequence produces 50 images in an imaging time of approximately 30 minutes. In these images, the signal from flowing blood appears dark against mediumgray myocardium and bright fat. Coronary arteries are seen as narrow dark structures with a disjointed appearance. Because of the motion of the heart and the tortuousness of the vessels, only small segments are visible on a given level at a given phase of the cardiac cycle. ${ }^{3}$

\section{MATERIALS AND METHODS}

The image reformatting technique uses a data set such as the cardiac images just described. Reformatting is performed by identifying the start of a vessel segment in one slice and locating the continuation of that vessel into a higher or lower slice. An equation of the plane connecting these features is determined and used to compute the location of each point in the new image. The reformatted image is built by scanning the new plane in a raster pattern at spacings equivalent to the 
Fig 1. Schematic illustration of the oblique plane description. Landmark features are identified by the user with a cursor on two or three image planes of the acquired 10-level data set. The angulated image is generated by three-dimensional linear interpolation.

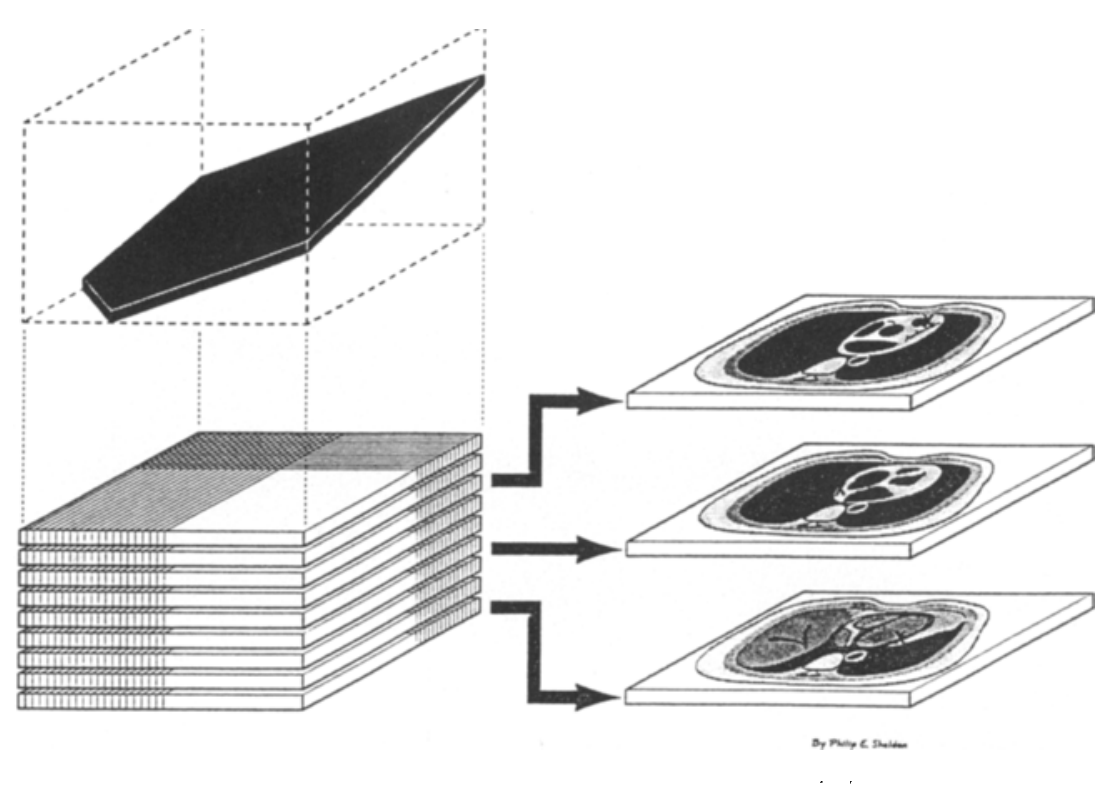

in-plane resolution of the original images (Fig 1). Rastering is done along lines parallel to the intersection of the oblique plane and the positive $y$ edge of the image space. This results in an oblique image orientation with the same top-bottom appearance as the original image. A linear interpolation is performed at each point by computing a weighted average of the eight surrounding neighbor pixels. The weighting is based on the proximity of each pixel used in the computation to the location of the new point. Because of the effective averaging of eight points to create the new value, the signal-to-noise ratio in the new image is noticeably improved. The greater the angulation away from the original orientation and into the lower-resolution slice direction, the greater the loss of resolution in the reformatted image. This can range from the in-plane resolution to the slice spacing, typically different by a factor of 10 . When small angulations are used, loss of resolution is not very noticeable. There are two methods for defining the plane. The user can place the cursor over three features of interest on multiple image levels to be included in the new oblique image. Alternatively, the user can draw a line and specify an angle for rotation of the new plane about the line. The view angle of the created image is normal to its surface, thus maintaining the size and aspect ratio of the object. A center of gravity computed from the three landmarks is used to center the oblique image. Once the plane is specified, parameters of the plane are stored for further use. Multiple parallel oblique planes with variable spacing can be requested by the user at the time that angulation is specified or a single oblique image can be created and later recalled to generate additional parallel slices. The reformatting procedure requires approximately 30 seconds per image when no array processor is used.

\section{RESULTS}

In this study, two contiguous levels showing left coronary artery anatomy were chosen, and coronary landmarks were noted on each slice. The reconstructed image appeared to connect elements of the same coronary vessel, which appeared as discontinuous segments on the two contiguous levels. In each of 16 cases examined, the visible length of coronary segments was increased with reformatting. Because of the chance of slice selection and angulation, no right coronary arteries and segments of only 20 vessels could be reconstructed, including 10 elements each of the left anterior descending (LAD) and left circumflex coronary arterial systems.

An example is illustrated in Fig 2. The two upper images are adjacent levels from the set of 10 originally acquired transaxial images. The image in (A) is the superior image of the two and shows the left main coronary artery and proximal LAD coronary artery. A distal segment of the LAD is identifiable in (B). Also visible is a segment of the left circumflex coronary artery. The operator marked with a cursor the left main coronary artery in (A) and the extensions of the distal circumflex and distal LAD coronary arteries in (B). The reformatted oblique image is shown in (C) and has combined the identified features of the two original images. The origin of the left main coronary artery is also more clearly seen.

A second example is shown in Fig 3. (A) and (B) again are from the originally acquired data set. The origin of the left main coronary artery extending into the proximal left circumflex coronary artery is visible in the superior image shown in (A). (B) shows a segment of the distal left 


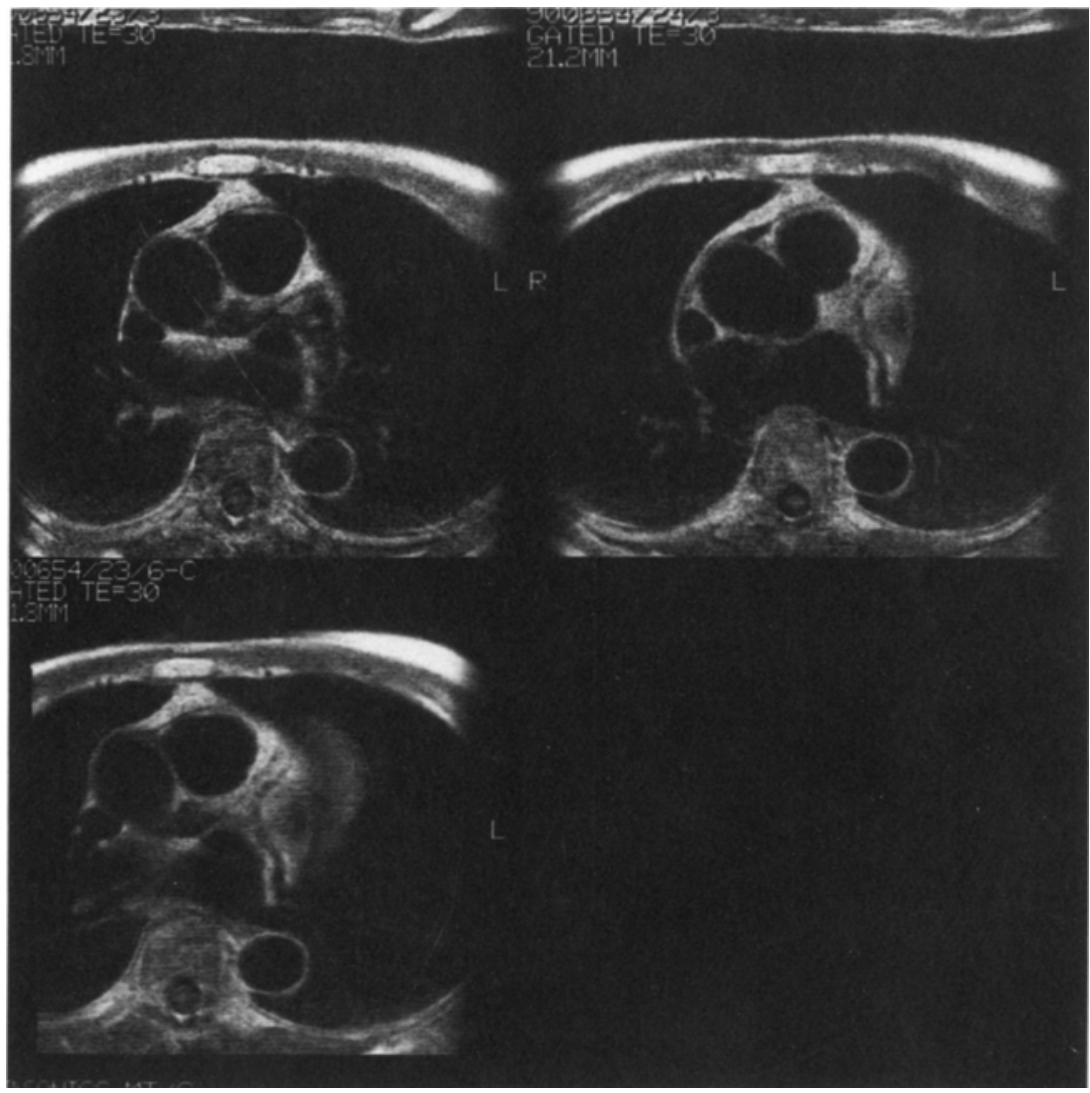

Fig 2. Two adjacent slices from the original data set (A and $B)$. The left main coronary artery and proximal LAD coronary artery are seen on the more superior image (A), while distal LAD coronary artery and a section of the left circumflex coronary artery are visible on the inferior image (B). These user-identified elements of both images are combined in the angulated view shown in the reformatted image (C). circumflex coronary artery. The reformatted oblique image shown in $(\mathrm{C})$ connects both regions of the left circumflex coronary artery, revealing a longer continuous segment than can be seen on either of the original images. The image edge seen cutting diagonally across the upper left corner results from the emergence of the oblique plane from the image space of the original data.

\section{DISCUSSION}

The appeal of this technique is that it is application driven; the specification of the oblique plane is determined by the presentation of the anatomy in the particular data set being examined. Small variations in anatomy between patients prevent the same degree of alignment accuracy using an oblique image acquisition. Multiple oblique acquisitions become prohibitive because of the accompanying increase in imaging time. This method of postprocessing is best used as a fine-tuning device and can be used in conjunction with any multislice data acquisition. In this particular application, reformatting was found to capture coronary anatomic detail not at all or only incompletely evident on the initial multiple level series of spin-echo images.

The success of this technique depends on the appearance of the coronary arteries in the original data set. Poor quality images for patients experiencing respiratory variation, arrythmia, or movement will prevent successful reformatting. Another limitation is the loss of spatial resolution that becomes unacceptable for large angulations. Images acquired by two-dimensional methods are generated by asymmetric voxels with typical in-plane pixel dimension to slice thickness ratios of $1: 10$. Reformatting an oblique plane with large amounts of angulation can result in a smeared appearance of the final image, with an in-plane pixel asymmetry ranging from the original in-plane pixel size to the slice thickness dimension. For small angulations, however (about $30^{\circ}$ or less), degradation of resolution is not very apparent.

A feature of the program allows the user to 
Fig 3. Left main coronary and proximal left circumflex arteries can be seen in the superior image ( $A$ ) of the acquired data set. A section of the distal left circumflex coronary artery is visible in the inferior (B) acquired image. Note that the resulting oblique image is centered on the area of interest as defined by the center of gravity

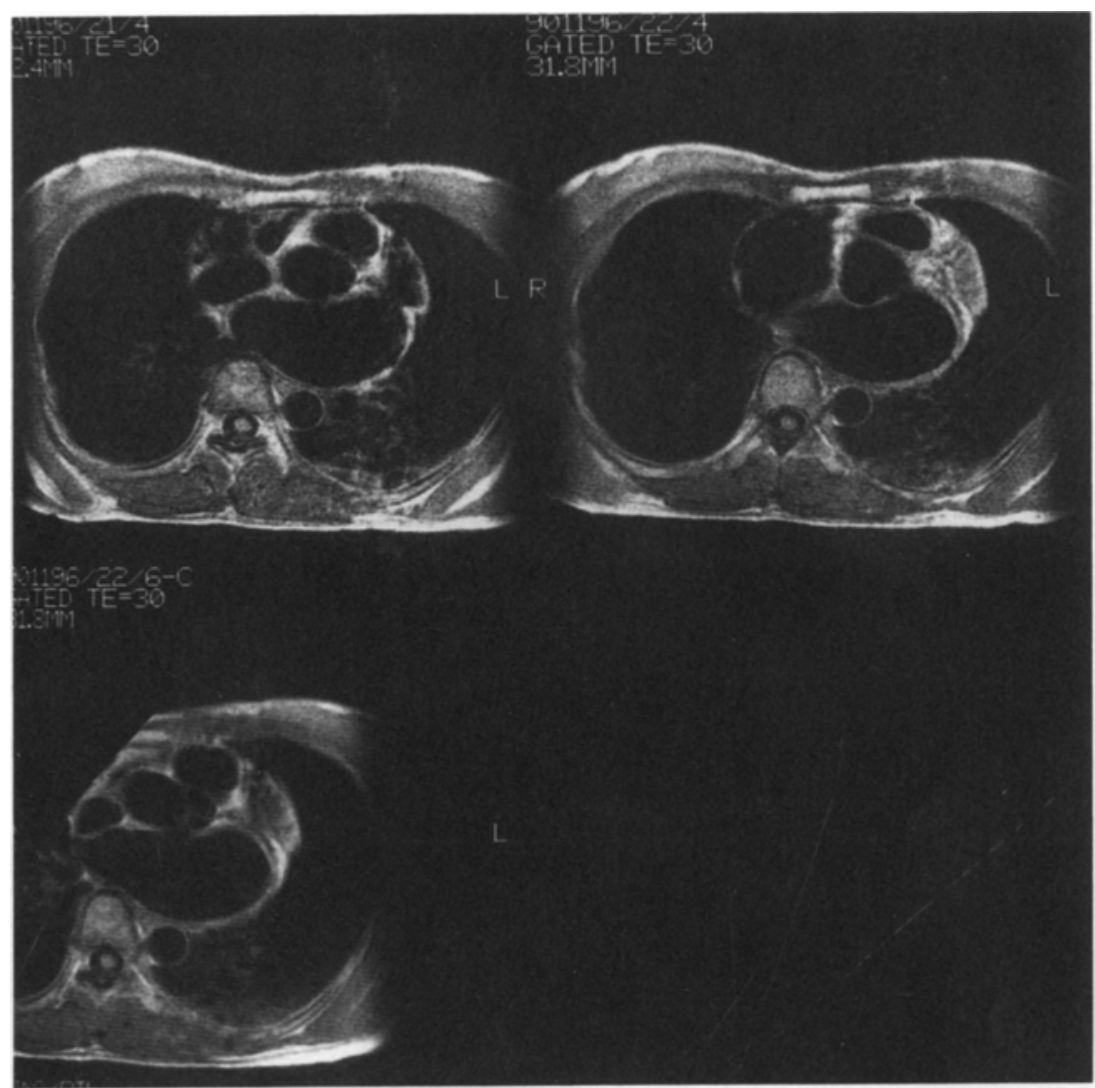
of the three landmarks.

define the desired angulation on a single cardiac phase image and request that it be automatically translated to all of the other cardiac phase images. This time series set of oblique images can be used in a cinematic display. This technique is not confined to coronary artery applications. It has been used successfully for reformatting images of the knee to better visualize the cruciate ligaments. It also has application in the optimal definition of the intervertebral disk and neural foramina.
Finally, data acquisition can be optimized for use with this technique. For example, an oblique orientation can be initially acquired perpendicular to the heart axis rather than the body axis. Flow compensation strategies can be used; sequences with finer resolution in both the sliceselect and in-plane directions and sequences with better coverage of the heart can be used. At this time, the reconstruction of nonplanar, ie, parabolic or cylindrical, surfaces are being investigated.

\section{REFERENCES}

1. Nishimura D, Macovski A, Pauly J: Coronary angiography by selective inversion recovery, in: Book of Abstracts. 7th Annual Meeting of the Society of Magnetic Resonance in Medicine. San Francisco, CA, 1988, p 726

2. White RD, Caputo GR, Mark AS, et al: Coronary artery bypass graft patency: Noninvasive evaluation with MR imaging. Radiology 164:681-686, 1987

3. Higgins CB: Overview of MR of the heart-1986. AJR 146:907-918, 1986 